\title{
Modular assembly of transposable element arrays by microsatellite targeting in the guayule and rice genomes
}

José A. Valdes Franco ${ }^{1,4}$, Yi Wang ${ }^{2}$, Naxin Huo², Grisel Ponciano², Howard A. Colvin ${ }^{3}$, Colleen M. McMahan², Yong Q. Gu${ }^{2 *}$ and William R. Belknap ${ }^{2}$

\begin{abstract}
Background: Guayule (Parthenium argentatum A. Gray) is a rubber-producing desert shrub native to Mexico and the United States. Guayule represents an alternative to Hevea brasiliensis as a source for commercial natural rubber. The efficient application of modern molecular/genetic tools to guayule improvement requires characterization of its genome.

Results: The $1.6 \mathrm{~Gb}$ guayule genome was sequenced, assembled and annotated. The final $1.5 \mathrm{~Gb}$ assembly, while fragmented $\left(N_{50}=22 \mathrm{~kb}\right)$, maps $>95 \%$ of the shotgun reads and is essentially complete. Approximately 40,000 transcribed, protein encoding genes were annotated on the assembly. Further characterization of this genome revealed 15 families of small, microsatellite-associated, transposable elements (TEs) with unexpected chromosomal distribution profiles. These SaTar (Satellite Targeted) elements, which are non-autonomous Mu-like elements (MULEs), were frequently observed in multimeric linear arrays of unrelated individual elements within which no individual element is interrupted by another. This uniformly non-nested TE multimer architecture has not been previously described in either eukaryotic or prokaryotic genomes. Five families of similarly distributed non-autonomous MULEs (microsatellite associated, modularly assembled) were characterized in the rice genome. Families of TEs with similar structures and distribution profiles were identified in sorghum and citrus.

Conclusion: The sequencing and assembly of the guayule genome provides a foundation for application of current crop improvement technologies to this plant. In addition, characterization of this genome revealed SaTar elements with distribution profiles unique among TEs. Satar targeting appears based on an alternative MULE recombination mechanism with the potential to impact gene evolution.
\end{abstract}

Keywords: Natural rubber, Genome, Assembly, Annotation, Class II transposable element, Non-autonomous, Transposon

\section{Background}

Guayule, a species in the Compositae (Asteraceae) family, is a perennial shrub native to the Chihuahuan Desert of North America that represents a potential commercial source of natural rubber [1]. There have been the numerous unsuccessful past efforts to develop guayule as a crop [2], however, both a narrow germplasm pool and the innate genetic complexity of guayule limited past breeding improvement efforts [1]. The importance of

\footnotetext{
* Correspondence: yong.gu@ars.usda.gov

${ }^{2}$ USDA-Agricultural Research Service, Western Regional Research Center,

Albany, CA, USA

Full list of author information is available at the end of the article
}

guayule, and other plants, as alternatives to Hevea brasiliensis as natural rubber sources can be appreciated when the economics and supply of Hevea rubber are considered. Seventy percent of worldwide rubber production (12.3 million metric tons in 2015) is utilized in tire fabrication [3], and natural rubber represents one of the largest corporate purchases made by this industry. The natural rubber supply is subject to both considerable price volatility and a number of issues associated with security of supply. For example, the Hevea tree is susceptible to South American Leaf Blight (SALB) caused by the endemic fungus Microcyclus ulei. SALB terminated commercial Hevea cultivation in 
South America in the early 1900's and remains a major threat to rubber production [4]. Thus, identification and development of alternative sources of natural rubber are important to the tire industry. The sequencing and annotation of the $1.6 \mathrm{~Gb}[5,6]$ nuclear genome of a diploid guayule was undertaken to facilitate the application of current molecular and breeding tools to guayule improvement.

The genome annotation process, which follows sequence acquisition and assembly, involves identification of encoded gene and structural components, essentially converting a compiled DNA sequence into an informationrich tool with broad applicability. The identification/ characterization of repeat sequences is a requisite first step for efficient and accurate genome annotation [7]. In most chromosomal domains, the repeated DNA sequences represent largely transposable elements (TEs) of two classes. The class I retrotransposons are mobilized through an RNA intermediate and make up large percentages of plant genomes [8]. The class II transposons mobilize through a DNA intermediates, and exist both in autonomous (TE encodes requisite transposase proteins) and non-autonomous (transposase function supplied in trans) forms [9]. Both TE types have non-random distribution profiles on plant chromosomes, with the class II TEs associated with gene-rich chromosomal regions [9]. The $\mathrm{Mu}$-like element (MULE) superfamily of class II TEs represents a large and diverse set of autonomous and non-autonomous elements that can make up a significant percentage of plant genomes $[10,11]$. The MULE TEs, particularly the Pack-MULEs, have been proposed as important mediators of plant gene evolution [12-15].

In the course of characterizing repeated sequences in initial guayule genome assemblies, a number of short, unrelated repetitive DNA sequences associated with microsatellites were identified. Microsatellites, or simple sequence repeats (SSRs), are regions of tandemly repeated short (1-6 bp) DNA repeats that are common features in genomes [16]. The guayule microsatelliteassociated elements were identified as microsatellitetargeted non-autonomous MULE elements by both structural and sequence similarities to guayule autonomous MULEs. While specific microsatellite-associated class II TEs [17-19], including autonomous MULE elements $[20,21]$, have been identified in plants, the elements in guayule had unique and unexpected features.

\section{Methods}

\section{Plant material}

All genomic and transcriptome sequences were derived from a single diploid guayule plant, accession W6 429 developed from a selection obtained in a 1942 Durango, Mexico collection expedition $[1,5,6]$.

\section{Sequence acquisition}

DNA was prepared as described previously [22], polyA-RNA was prepared employing Qiagen RNeasy/ QIAshredder protocols.

Illumina (300 bp paired-end) shotgun sequencing libraries were made using the Kapa Biosystems protocol: High-Throughput NGS LibraryPreparation Technical Guide for Illumina TM platforms (KR0427 - V1.12) and sequenced with MiSeq ${ }^{\circ}$ Reagent Kit v3 Reagents on an Illumina MiSeq. The Roche 454 sequencing libraries were prepared using the Rapid Library Preparation Manual for GSFLX+ and GS Junior + Series (May 2011) and sequenced with Roche 454 GSFLX +. The Illumina matepair libraries with insert sizes of 500, 700 and $1 \mathrm{~kb}$ were made following the Nextera ${ }^{\circ}$ Mate Pair Library Illumina HiSeq 2500.

Transcriptome sequence was obtained from polyARNA libraries constructed following the Kapa Biosystems protocol: KAPA Stranded mRNA-Seq Kit for illumina ${ }^{\circ}$ platform (KR0960 -v3.15) and sequenced on an Illumina HiSeq $2500\left(10^{8} \times 150 \mathrm{bp}\right.$ reads). Greater than $80 \%$ of the transcriptome reads mapped to the Meraculous assembly.

\section{Meraculous and CLC Genomics Workbench genome assemblies}

Meraculous is a whole genome assembler for next generation sequencing data geared for large genomes [23]. The guayule genome was first assembled using the Mercaculous assembler with the Illumina reads (shotgun and Nextera). This assembly encompassed $1.5 \mathrm{~Gb}$ $\left(\mathrm{N}_{50}=22 \mathrm{~Kb}, 260 \mathrm{k}\right.$ Scaffolds $)$ and mapped $>95 \%$ of the shotgun reads. CLC Genomics Workbench assembly (version 8.5.1, in conjunction with scaffolding using SSPACE version 2.0 [24]) employed the Illumina and Roche 454 reads and provided improved representation of simple sequence repeat domains. This assembly encompassed $0.9 \mathrm{~GB}\left(\mathrm{~N}_{50}=28 \mathrm{~Kb}, 59 \mathrm{k}\right.$ Scaffolds $)$ and retained $>95 \%$ of the annotated genes.

\section{Transcriptome assembly}

For annotation purposes, the transcriptome was assembled employing Cufflinks [25] and Trinity [26] assemblers, and further processed with PASA [27].

\section{Gene annotation}

Genome repeat sequences were annotated with RepeatMasker. Approximately 40,000 protein-encoding, transcribed, genes were annotated (Trinity/MAKER/Cufflinks) $[25,26,28]$ on the Meraculous assembly. Genome repeat sequences were annotated denovo with RepeatMasker (http://www.repeatmasker.org/webrepeatmaskerhelp.html). Additionally, Augustus gene prediction software (http:// bioinf.uni-greifswald.de/augustus/) was employed to identify expressed genes on the 33 guayule scaffolds artificially 
assembled in Additional file 1. Expressed genes in this assembly were verified by BLASTp returns with $E$ values less than $\mathrm{e}-15$.

\section{Computational de novo identification of SaTar elements} Software designed to identify potential Satellite Targeted (SaTar) elements in any genome was employed. Entered scaffolds are scanned for repeated sequences (250-800 bp in length, $\geq 20 \% \mathrm{GC}$ content) defined by TA microsatellite domains ( $\geq 12 \mathrm{bp}$ in length). The program utilizes MISA to identify microsatellite domains (http://pgrc.ipkgatersleben.de/misa/misa.html). Software and Users Guide available at: http://probes.pw.usda.gov/Guayule.

\section{Results}

\section{Sequencing, assembly and annotation of the guayule genome}

The genes annotated from a single artificial $16 \mathrm{Mb}$ assembly (approximately $1 \%$ of the genome) composed of the 33 longest Meraculous scaffolds is shown in Fig. 1a. The locations of the 505 annotated genes are indicated, a gene density consistent with predictions based on the overall genome (Table 1). The observed non-genic domains in this assembly are composed largely of retrotransposon and unclassified interspersed repetitive sequences (Table 1). However, no evidence for recent, widespread, amplification of specific Class I or Class II transposon/ retrotransposon families was found in either the guayule assemblies or the collected reads. Syntenic analysis of the genes encoded on individual scaffolds was consistent with two rounds of genome-wide duplication in the last 40 MY [29].

This Meraculous assembly allows characterization of particular genes of interest for guayule improvement, including those involved in rubber biosynthesis. For example, Fig. 2 shows the structures of genes encoding proteins associated with a selected portion on the mevalonate sourced rubber biosynthetic pathway. In general the Meraculous assembly contains the expected isoforms/structures of these genes [30, 31], and encode mRNAs specifically associated with guayule rubber biosynthesis (SRPP-1, Fig. 2) [32].

\section{Structure of guayule gSaTars}

In the course of characterizing the repetitive DNA content in the guayule genomic scaffolds, in particular characterization of non-autonomous Class II TEs, an unexpected pattern emerged. When scaffolds were probed with libraries of small $(\leq 1.7 \mathrm{kbp})$ terminal inverted repeat elements, clusters of the elements were frequently observed. These clusters had two unexpected properties. First, they were largely associated with microsatellite domains such that individual elements within the cluster are flanked by simple sequence repeats, often $(\mathrm{TA})_{\mathrm{n}}$. Second, within the clusters individual elements were modularly assembled, in no case did one microsatellite-flanked element interrupt another. This architecture suggested that the observed modular assembly resulted from the specific targeting of these sequences to microsatellite domains $[17,18,20,21]$. For this reason, these elements are referred to as SaTars, or Satellite Targeted, transposable elements.

The guayule SaTars, or gSaTars, described here range in size from approximately 400 to $1700 \mathrm{bp}$, depending on the individual family (Table 2, Additional file 1). As shown in Fig. 3, they have several structural properties common to non-autonomous class II TEs. The gSaTars are of appropriate size and are defined by imperfect terminal inverted repeat (TIR) domains (Table 2). Eleven of the fifteen gSaTar families contain individual members not localized to microsatellites and flanked by 10 bp target site duplications (TSDs) (Fig. 3, Table 2). In contrast to standard non-autonomous Class II TEs however, a subset of the gSaTars appear preferentially targeted to microsatellite domains (Table 2). Probing (BLAST) the genome with either form (TSD or microsatellite targeted) 
Table 1 Genes and repetitive DNA on the 1.5 Gb Meraculous assemly

\begin{tabular}{llll}
\hline Mapped transcripts & Number & & \\
CDS & 268,632 & cdhit uniques 25,740 \\
mRNA & 61,170 & & \\
Full length ORF & 44,750 & & \\
Refseq db & 42,708 & & \\
Filter > 80\% & 39,347 & & \\
GO (swisprot 90\%) & 23,144 & & Percentage \\
Repetitive content (denovo) & Number & Content (bp) & \\
LTR & 439,305 & $368,003,201$ & $24.18 \%$ \\
LINEs & 51,983 & $28,517,926$ & $1.87 \%$ \\
SINEs & 15,372 & $3,504,610$ & $0.23 \%$ \\
DNA elements & 196,517 & $51,599,240$ & $3.39 \%$ \\
Unclassified & $1,030,342$ & $355,313,348$ & $23.35 \%$ \\
Total interpersed repeats & & $806,938,325$ & $53.02 \%$ \\
Small RNA & 644 & 84,033 & $0.01 \%$ \\
Satellites & 535 & 308,909 & $0.02 \%$ \\
Simple repeats & 33,165 & $50,184,037$ & $3.30 \%$ \\
Low complexity & 44,121 & $2,189,640$ & $0.14 \%$ \\
\hline
\end{tabular}

returns equally elements with both types of insertion events.

While the gSaTar elements predominantly localized to $(\mathrm{TA})_{\mathrm{n}}$ microsatellite domains, in guayule the type and complexity of the microsatellite domains flanking gSaTar elements are highly variable (Fig. 4, for example). Among the gSaTar families, at least $78 \%$ of the elements are flanked by either microsatellite domains or TSDs (Table 2). The remaining elements, i.e. those with neither architecture, appear to represent loci in which terminal and/or flanking sequences have been altered by local deletions and/or rearrangements.

In Fig. 4, individual members of four gSaTar families flanked by either TSDs or microsatellite domains are aligned. In cases where the gSaTar elements are flanked by identical microsatellite domains, both structures are consistent with derivation from a straightforward "cut and paste" transpositional mechanism resulting in a 10 bp TSDs. However, as indicated in Fig. 4, the microsatellite sequences immediately flanking the elements are often inconsistent with this mechanism.

The relative frequency of the different gSaTar families in the genome are highly variable, and can be estimated

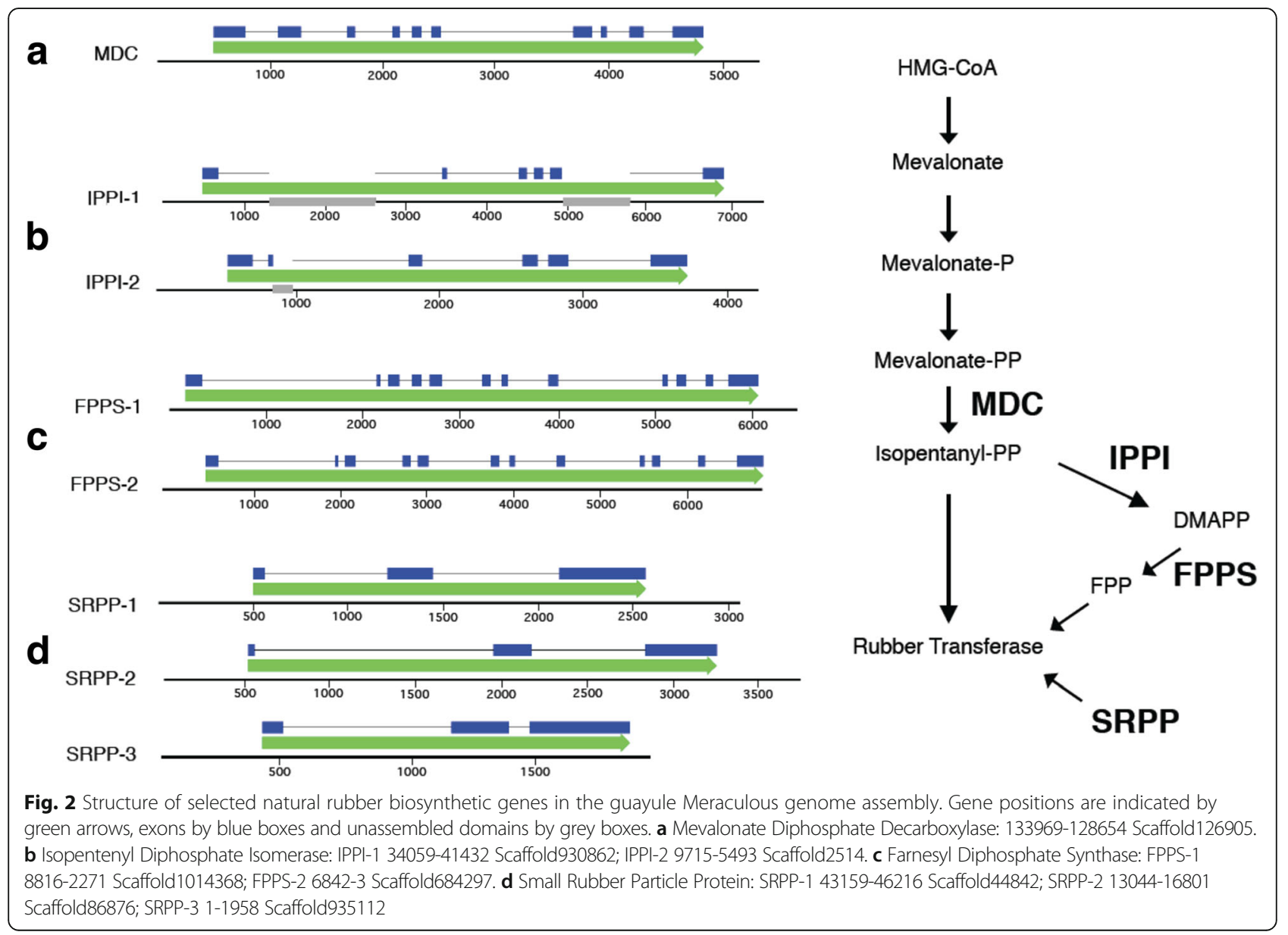


Table 2 Architecture and distribution of guayule gSaTar elements

\begin{tabular}{|c|c|c|c|c|c|}
\hline \multirow[t]{2}{*}{ gSaTar element } & & \multirow{2}{*}{$\begin{array}{l}\operatorname{TIR}^{\mathrm{a}} \text { length } \\
\mathrm{bp}\end{array}$} & \multirow{2}{*}{$\begin{array}{l}\text { Flanked } \\
\text { microsatellite } \\
\%\end{array}$} & \multirow{2}{*}{$\begin{array}{l}\text { Flanked TSD }{ }^{\mathrm{b}} \\
\%\end{array}$} & \multirow{2}{*}{$\begin{array}{l}\text { Linked/Fusec } \\
\text { gSaTar } \\
\%\end{array}$} \\
\hline & & & & & \\
\hline gSaTar1 & 990 & 55 & 77 & 6 & 31 \\
\hline gSaTar2 & 400 & 40 & 70 & 22 & 19 \\
\hline gSaTar3 & 425 & 170 & 77 & 10 & 22 \\
\hline gSaTar4 & 400 & 150 & 95 & 4 & 28 \\
\hline gSaTar5 & 1050 & 60 & 80 & 20 & 20 \\
\hline gSaTar6 & 500 & 60 & 90 & 4 & 33 \\
\hline gSaTar7 & 400 & 50 & 74 & 15 & 21 \\
\hline gSaTar8 & 440 & 120 & 87 & 7 & 29 \\
\hline gSaTar9 & 520 & 60 & 90 & 0 & 20 \\
\hline gSaTar10 & 530 & 55 & 91 & 0 & 27 \\
\hline gSaTar11 & 1030 & 60 & 78 & 0 & 26 \\
\hline gSaTar12 & 380 & 180 & 93 & 3 & 47 \\
\hline gSaTar13 & 410 & 160 & 94 & 0 & 38 \\
\hline gSaTar14 & 1120 & 250 & 68 & 14 & 37 \\
\hline gSaTar15 & 750 & 55 & 62 & 24 & 22 \\
\hline
\end{tabular}

${ }^{\text {a Terminal Inverted Repeat }}$

${ }^{\mathrm{b}}$ Target Site Duplication

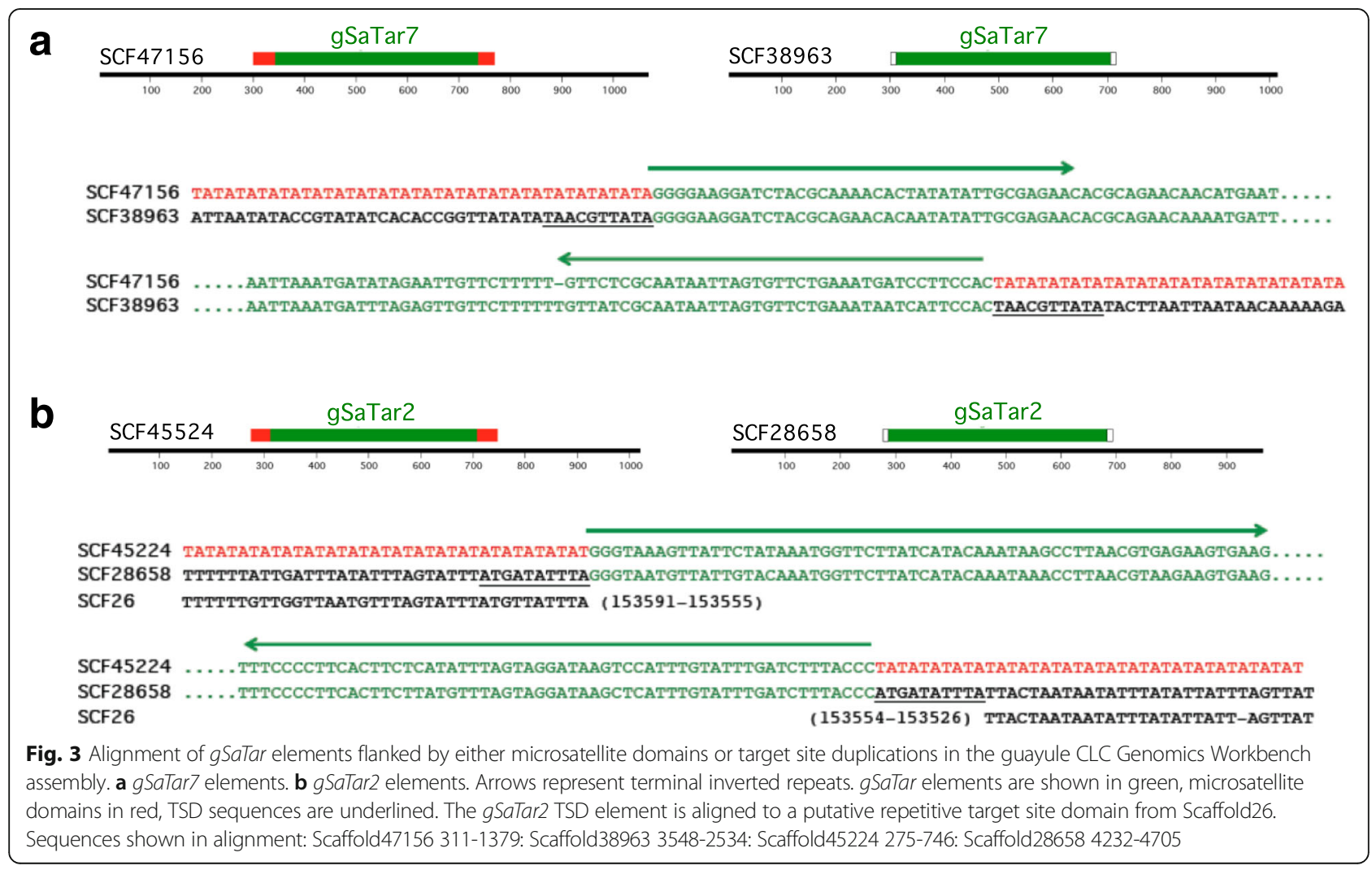




\section{gSaTar2}

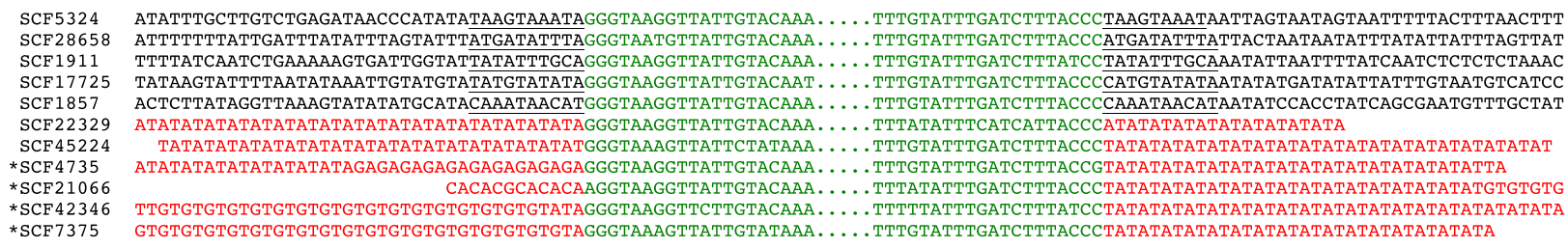

\section{gSaTar4}

SCF25664 ACTAAGAAGGGATGCCAACAAAAATGAATAAAAGCCATGGTTTAGGTTCGTGTACAAA. . . . TTTGTACACTAACCTTACCCTAAAAGCCATAACAGCAACAATGTTAAACGAATAAAAGCC 促 SCF 8568 CTTTTATTCGTTTAACATTGTTGCTGTTATGGCTTTTAGGGTAAGGTTAGTGTACAAA . . . . TTTGTACACGAACCTAAACCATGGCTTTTATTCATTTTTGTTGGCATCCCTTCTTAGTTC SCF976 TGCACTGCCATGTCATTTATAACCTCAACTATCCTTAAGGGTGAGGTTAATGTACAAA.... TTTGTACACGAACCTAAACCCTATCCTTAAGCCACTTAATCACCGGTTGCACTCCATCCA

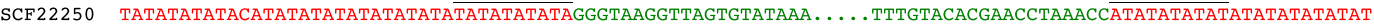

SCF10118 TATATATATATATATATATATATATATATATATATATATGGTAAGgTTATTATACAAA . . . TTTGTACACGAACCTAAAC-ATATATATATATATATATATATATATATATATATATATAT TATATATATATATATATATATATATATATATATATATAGGGTAGGTTAGTGTACAAA $\ldots \ldots$ TTTGTACACGAACCTAAAC-ATATATATATATATATATATATATATATATATATATATAT * $\mathrm{SCF} 683$

*SCF22962

* SCF 3653

*SCF 19755

*SCF 35289 TATATATATATATATATATAGGTTGAGATTAGTGTACAAA. GTGTGTGTGTGTATGTGTGTGTGTGTGTGTGGGGGTGAGGTTAGTGTACAAA. . TATATATATATATATATATATATATATATATATATATAGGGTAAGGTTAGTGTACAAA. . TTTGTACAGGAACCTAAAC-ACACACACACACACACACACACACACACATATATATATATA TTTGTACACGAACCTTTATCTATATATATATATATATATATATATATATATATATATATAT TTTGTACACGAACCTAAAC-ACACACACACACACACACACACACACA TTTGTACACTAATCTTACCCTATATATATATAT

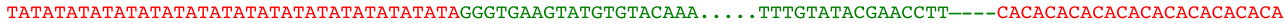

\section{gSaTar7} (1) T. 10633 T... T. 50193 GTA SCF 7156 TATATATATATATATATATATATATATATATATATATATAGGGGAAGGATCTACGCAAAA....... TTCTGAAATGATCCTTCCACTATATATATATATATATATATATATATATATA SCF 32036 TATATATATATATATATATATATATATATATATATATATAGGGGAAGGATCTACGCAGAA....... CTCTGAAATGATCCTTCCACTATATATATATATATATAT

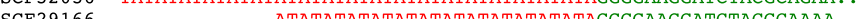
*

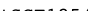
* $\mathrm{SCF} 1254$ * SCF 3269 *SCF19831

*SCF 47979 *SCF11908 *SCF 36668 ATRT: DTATATATATATATATATATATGCATATATAGGGGAMGATCTATGCAGAA. . ATATATATATATATATATATATATGTATATAGGGGAAGGATCTACGCAGAA. TATATATATATATATATATATATATATAGGGAA-GGATCTACGCAGAA. ACACACACACATATATATATATATATATATATATATATAT-GGGAAGGATCTACGCAGAA CTATCTATCTATCTATCTATCTATCTATCAATATCATATAGGGGAATGATCTACATAGAA ACTATATATATAGGGGAaGGATCTACGCAGAA. ATAAATAATTAAATAAATAAATAAATAAATAGGGGAAGGATCTACGCAGAA . .

TTCTGAAATGATCCTTCCACTATATATATATATATATATATATATATATATATATATATA TTCTGAAATGATCCTTCCGCCACACACACACACACACACACACATACACACACACACACA .TTCTGAAATGATCCTTCCACTATGTGTATGTGTGTGTGTGTGTGTGTGTGTATGTG TTCTGAAATGATCATTCCACTACACACACACACACACACAC

.TTCTGAAATGATCATTCCATTATATGTATGTATGTATGTATGTATGTATGTATG TTCTGAAATGATTCTTCCACGTATCTCACACACACACACACACACACACACACACACACA
TTCTGAAATGATCCTTCCACTACACACACACACACACAC

\section{gSaTar15}

$\mathrm{SCF} 1$

SCF 4912

SCF 5176

SCF5 2660

SCF 2459

SCF 41110

SCF 1804

SCF 1804

SCF 5040

*SCF 26365
.TTTTTAGGGGATCTCTCCTCTAGGAATAACTACCATTGTAACCAACACATCCTTTCAATC TGTACTGATACATATTTAGAAAATGGCTATTAGGAATAACGGTTAGGATCAAATAAAAA AGATATGTTTATGAGATTTTATAAGAAGATACTTAACAGGGGTTAGGATCAAATAAAAA ATATTATATTATATAGTGTTATGATTAAATAAAAA.

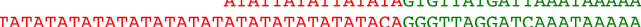
TATATATATATATATATATATATATATATATATATATATAGGGTTATGATCAAATAAAAA . . TATATATATATATATATATATATATATATATATATATATA--GTTAGGATCAAATAaTAa. ATATATATATATATATATATATATATATATATATATATAT-GGTTMGGATCABATMAABa. . .

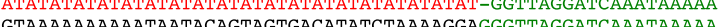
TATATATATATATATATATATATATATATATATATATATAGGGTTAGGATCAAATAAAAA TATATATATATATATAGAGAGAGAGAGAGAGAGAGAGAGGGGGTTAGGATCAAATAAAAA . .
. TTTTATCGGATCTCACCCCATAAACGTATAACTAGTACCACAAATTTGACTTGAACGAT TTTATAGgGGATCTCTCCCCTATATATATATATATATATATATATAT

TTTTTAGGGGATCTCTCCCCTATATATATATATATATATATATATATAGATACACACACA

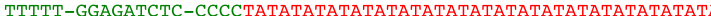
. TTTTAAGG-GATCTCTCCCTTATATATATATATATATATATATATATATATATATA

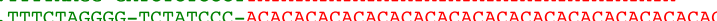
TTTTTAGGGGATCACTCCC-TATATATATATAT

t

Fig. 4 Alignment of gSaTar element termini and flanking regions. gSaTar elements are shown in green, microsatellite domains in red, TSD sequences are underlined. The asterisk indicates a flanking structure inconsistent with a 10 bp target site duplication on insertion

by analysis of their distribution on the $16 \mathrm{Mb}(1 \%$ of genome) artificial assembly (Fig. 1a). This assembly contains 215 gSaTar elements, 32\% of which are linked to other gSaTars (Additional file 2) indicating the presence of over 20,000 total elements constituting approximately $1 \%$ of the guayule genome.

\section{Genomic distribution of gSaTar elements}

The distribution of gSaTar elements along the guayule scaffolds is strikingly different from MITEs and other non-autonomous Class II TEs. MITEs are associated with gene-rich domains in the genome [33, 34]. Specific targeting of MITE insertion events to elements of the same, and different, MITE families results in clusters of these elements [35]. The gSaTar elements are also found in clusters on the guayule scaffolds, but with frequency and architecture strikingly different from described in MITEs. In terms of frequency, the gSaTar elements are localized to clusters more frequently than observed with the most commonly associated rice MITE elements (Table 2) [35]. And in contrast to the rice-MITE clusters where insertion of MITEs into existing elements is more commonly observed than adjacent insertion [35], the gSaTar clusters are always assembled modularly, with intact individual elements linked via microsatellite domains. Examples of gSaTar clusters are shown in Fig. 5 (selected cluster domain sequences in Additional file 3). In these clusters, elements from the different families are assembled by adjacent insertions flanked by microsatellites. Within the guayule gSaTar clusters internal deletions are observed, some of which remove the intervening satellite sequence in addition to gSaTar element ends resulting fusion of elements within the cluster (Fig. 5h and i, for example). However, in cataloging over $300 \mathrm{gSaTar}$ clusters, not a single case of one gSaTar element interrupting another has been 


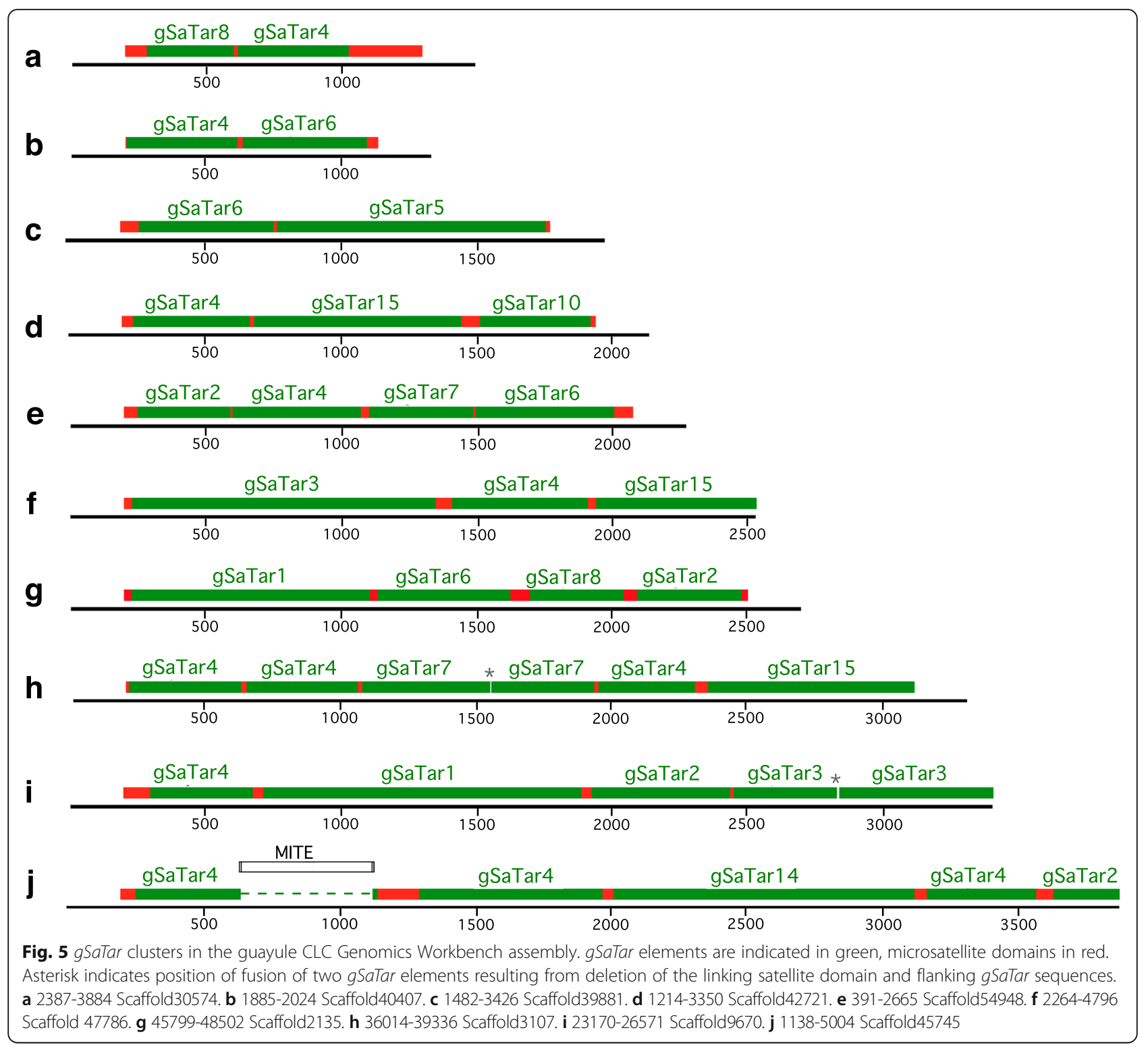

observed, supporting a mobilization mechanism distinct from that involved in the expected non-autonomous Class II TE transposition.

Analysis of one of the gSaTar clusters (Scaffold23207) revealed a large, low copy, TA-satellite-linked, repetitive DNA at one end (Additional file 4a). Further characterization indicated that this sequence represents a terminal fragment of a larger defective autonomous gSaTar-MULE1 (AgS-MULE1) $M u$-like transposon [10], an apparently full length copy of which occurs on scaffold 15495 (Additional file 4b). This $5.3 \mathrm{~kb}$ MULE element is defined by 400 bp TIRs. A BLAST search of the guayule CLC assembly with the autonomous MULE TIRs returned 5 hits, and in all cases the terminal TIRs were flanked by microsatellite domains. Five additional Autonomous gSaTar-MULEs have been identified in the guayule assemblies (Additional file 1). As indicated (Additional file 5), the TIRs defining four of these families are similar to those defining previously described gSaTar families (Additional file 1). Both the definition of gSaTar elements by long TIRs similar to the AgS-MULE elements described above, and the identification of individual members of eleven of the fifteen families flanked by $10 \mathrm{bp}$ TSDs in complex sequence rather than microsatellite domains [36], indicate that the gSaTar elements described here represent non-autonomous MULEs that have been specifically targeted to microsatellite domains.

\section{SaTar elements in the rice genome}

In order to determine if the SaTar mobilization pathway is a general feature of plant genomes, a specific search algorithm for identification of repetitive DNA 
sequences flanked by microsatellite domains was developed (Methods section). The complete genomic assemblies of rice (Oryza sativa (japonica) v7_JGI, [37, 38]) was evaluated employing this algorithm. Five families of rice SaTars (rSaTars) were returned by the algorithm (Table 3). While the SaTar elements are considerably less frequent in rice as compared to the guayule genome (Table 3, Fig. 1b), the rSaTar and gSatar elements contain a number of common features. Similar to the gSaTars, the $r S a T a r$ families range in size from 380 to 1900 bp. Two resources were employed in the classification of these elements, the RiTE rice transposable element database [36] and a comprehensive listing of rice MULE elements [39]. Four of these families were identified as non-autonomous MULE or Mutator-MITE elements, the most abundant rice TE type (Additional file 6). By comparison to previously mapped rice MULE elements [39], rSaTar1, 3 and 5 were further characterized as nonPack-MULEs. While the diverse MULE element families in the rice genome are largely flanked by 9 bp TSDs [39], only the rSaTars in Table 2 were flagged as microsatellite associated by the search algorithm. The final element, rSaTar2, was previously identified as small MITE-like mobile elements targeted to (TA) $n$ microsatellites (Micron) in rice [17]. However, as shown in Additional file 7, the TIR domains of the rSaTar2/Micron elements are highly similar to the TIRs of an autonomous $M u$-like transposon found on the chromosome one of the Oryza sativa (japonica) genome, indicating that this element is also a non-autonomous rice MULE.

These rSaTar elements share common features with the guayule gSaTars described above (Table 2). First, the rSaTar 1,2 and 4 families are defined by TIRs. Second, the elements were frequently associated with microsatellite domains (Table 3). Similar to the four of the gSaTar families, the rSaTar1, rSaTar2, rSaTar4 and rSaTar5 families had no members flanked by TSDs. In contrast, among the $r S a T a r 3$ elements, this structure was dominant. In addition, the rSaTar elements were frequently clustered (Fig. 6, Additional file 8). For example, of the 36 rSaTar1 elements in the rice genome, 6 are linked to rSaTar2 elements via microsatellite domains (Fig. 6). Of the $r$ SaTar2 elements, 27 are linked to rSaTar4 elements (Additional file 8). Finally, in none of the clusters, containing either a single or multiple $r$ SaTar types, does one $r$ SaTar element interrupt another.

In addition to these non-autonomous rSaTar elements, four defective autonomous MULEs which appear to have been mobilized as rSaTars (Autonomous rSaTar-MULEs, ArS-MULEs) have been identified in the rice genome (Additional file 9). Again similar to the guayule architecture, other $r S a T a r$ elments are found in the satellite domains flanking these autonomous TEs (Additional files 9 and 10). Of the 12 ArS-MULE1 elements, 6 are linked to $r S a T a r 2$ elements.

In addition to the Oryza sativa ssp. japonica assembly, the availability of a draft genome from the second cultivated subspecies or varietal group, Oryza sativa ssp. indica (assembly ASM465v1 [40]) facilitates identification of potential rSaTar element insertion sites. These groups diverged relatively recently (approximately 0.44 MYA [41]). Of the 51 rSaTar2 sites on japonica chromosome 1, 9 are conserved on the indica assembly (Additional file 11). In another 20 cases, however, these japonica insertion sites are represented by unoccupied TA microsatellite domains of variable length in the indica assembly (Fig. 7, Additional file 12). The remainder of the japonica rSaTar2 insertion domains are either deleted or unassembled in the indica genome. A similar relative $r$ SaTar3 insertion into an empty satellite flanking an $r$ SaTar1 element on japonica chromosome 3 is shown in Fig. 8. The presence of both conserved and non-conserved rSaTar2 loci in japonica and indica indicates that the SaTar targeting system was active before and after the divergence of these two subspecies/varietal groups.

SaTar-like elements with structures and distribution profiles were also identified in the sorghum (PhytozomeV9.0:Sbicolor_79 [42], Additional files 13 and 14).

Three families of sorghum SaTars (sSaTars) were identified employing the algorithm described above (Additional file 13). All three sSaTar elements are small (233-373 bp) MITE-like TIR elements. Similar to rSaTarland 2, no sSaTar-like elements defined by TSDs were found in the sorghum genome. As indicated in Additional file 13, there are a total of 1261 of the $s$ SaTar elements on the sorghum genome, leading to on approximately one element every $600 \mathrm{~kb}$ (1261 elements on $730 \mathrm{mb}$ ). However, the clustering of these elements is

Table 3 Architecture and distribution of rice rSaTar elements

\begin{tabular}{llllll}
\hline rSaTar element & Oryza sativa v7_JGl & Size bp & Total elements & Flanked microsatellite & Flanked TSD $^{a}$ \\
\hline rSatar1 non-Pack-MULE & Chr2 9609252-9609849 & 600 & 36 & 33 & 0 \\
rSaTar2 Micron & Chr2 16620771-16621153 & 380 & 510 & 492 & 0 \\
rSaTar3 non-Pack-MULE & Chr1 6228684-6229066 & 380 & 224 & 41 & 155 \\
rSaTar4 & Chr10 17637383-17638698 & 1500 & 176 & 153 & 0 \\
rSaTar5 non-Pack-MULE & Chr12 21079988-21081881 & 1900 & 5 & 5 & 0 \\
\hline
\end{tabular}

${ }^{\text {TTarget Site Duplication }}$ 


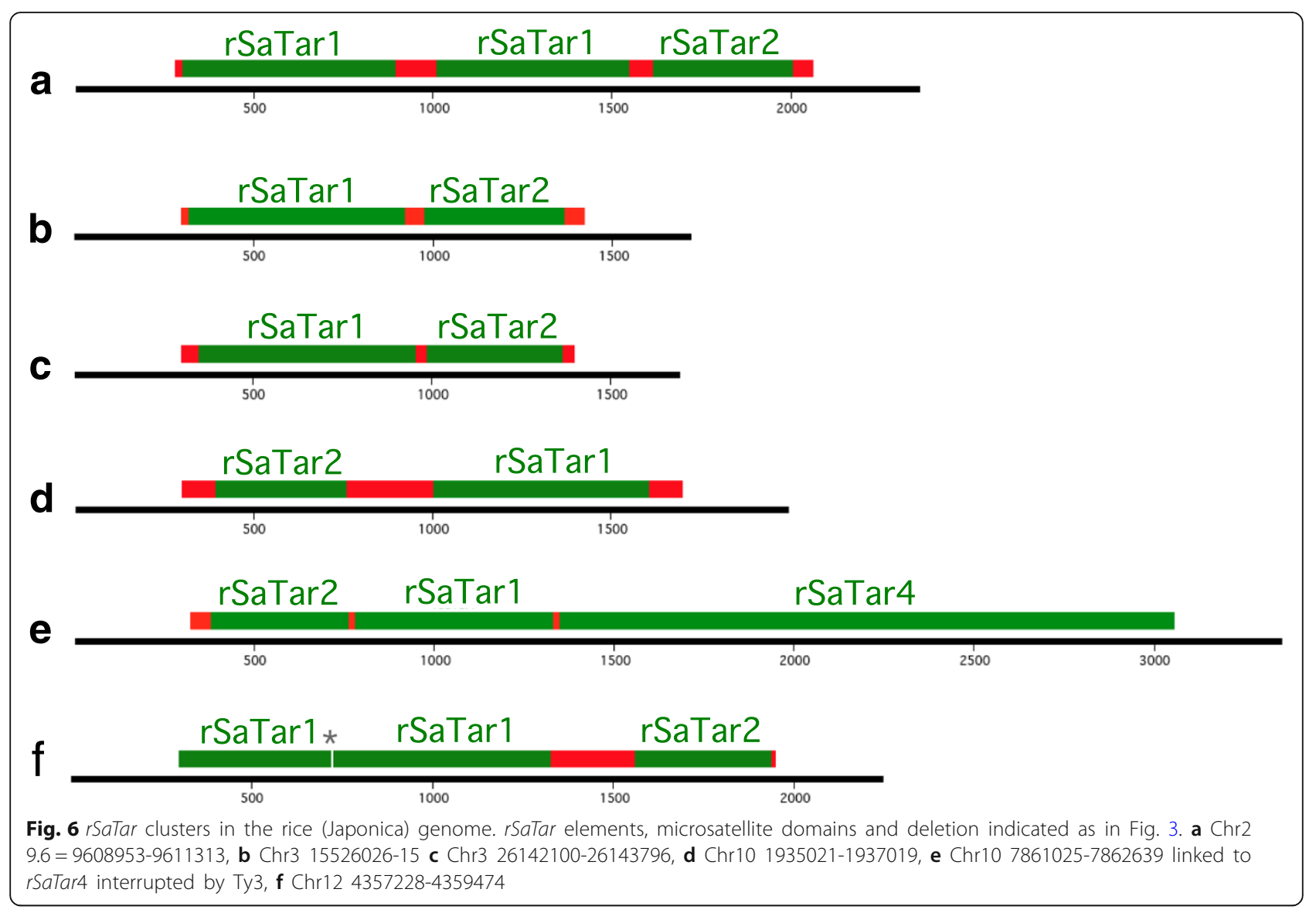

clearly shown in Additional file 14, which shows a cluster of 8 elements on chromosome 1. Degree of clustering of the sSaTar elements (Additional file 13) is similar to the guayule gSaTar elements (Table 1).

The algorithm employed to identify SaTar elements in rice and sorghum failed to return elements from the citrus genome (Cclementina_182_v1) [43]. However, a manual search of sequences flanking TA satellite domains resulted in identification of three families of small citrus SaTars (cSaTars) (Additional file 15). The cSaTar elements are similar to the guayule, rice and sorghum elements of (definition by TIRs, clustering on chromosomes, modular assembly). In addition, while the majority of these elements are flanked by microsatellite domains, each family contains members flanked by 9 or 10 bp TSDs (Additional file 15). However, the $c S a T a r$ elements appear relatively ancient as compared to the gSaTar and rSaTar elements. Examples of cSaTar clusters are shown in Additional file 16. Finally, in no cases did the sSaTar or the $c S a T a r$ elements within clusters interrupt each other.

\section{Discussion}

The successful development of guayule as a commercial source of natural rubber is dependent upon improvement of both its agronomic properties and rubber yield.
To this end, the sequencing, assembly and annotation of the guayule genome was undertaken to promote the application of current molecular and breeding tools improvement efforts. While fragmented (260 k Scaffolds), the Meraculous assembly reported here is essentially complete allowing, for identification and utilization of specific genes and gene components, as well as contributing to the development of molecular breeding tools (such as genotyping-by-sequencing [5]).

\section{Guayule gSaTar elements are MULE TEs}

The gSaTar elements were identified in the guayule genome as families of microsatellite-defined, non-autonomous MULE elements with a unique and unexpected chromosomal distribution profile. The MULE designation is based upon structural features of the gSaTar elements, characterization of insertion sites and TIR sequence similarity to autonomous guayule MULE transposons.

The gSaTar elements are similar to MULE elements in terms of size and structure (defined by TIRs) (Fig. 3, Table 2) [10]. Previously described non-autonomous MULE elements are flanked by 8-11 bp TSDs generated during the "cut and paste" transpositional process [15]. Most of the non-autonomous gSatar families described here (Table 2) have individual members flanked by $10 \mathrm{bp}$ 


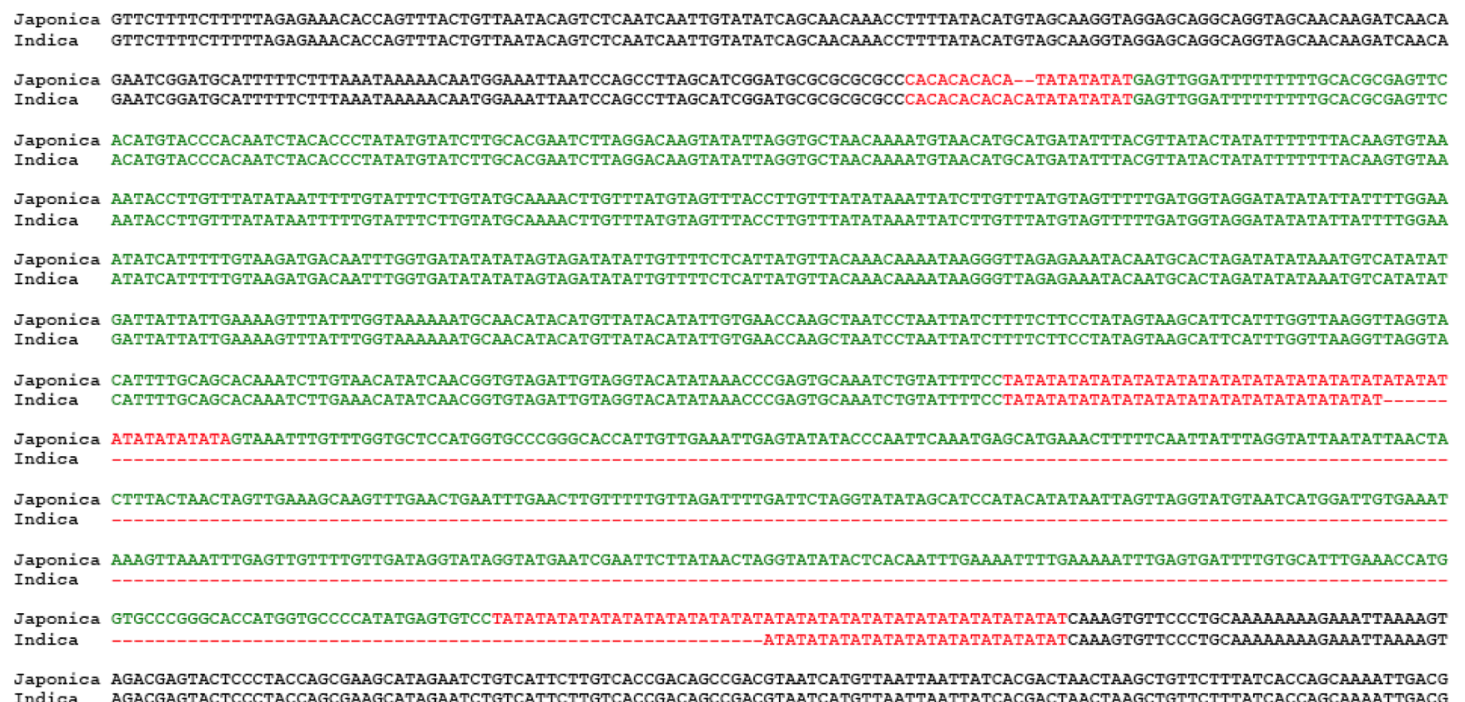

Fig. 7 Alignment of rice chromosome 3 sequences indicating an rSaTar2 insertion in Japonica relative to Indica. Microsatellite domains are indicated in red, gSaTar domains are indicated in green. Japonica sequence from Oryza sativa (japonica) v7_JGl 15526130-15527603, Indica from Oryza sativa (indica) assembly ASM465v1 17276496-17277536

TSDs, indicating that these elements are capable of mobilization through a similar pathway. The designation of non-autonomous gSaTar elements as MULEs is also supported by TIR sequence similarity to autonomous, microsatellite-targeted, AgS-MULE elements that are similarly targeted to microsatellite domains (Additional files 1,4 and 5). This sequence similarity indicates that the non-autonomous gSaTars are mobilized by AgSMULE transposase activities supplied in trans.
However, previously described autonomous and nonautonomous MULE elements do not display the sequencespecific (microsatellite domain) targeting exhibited by the gSaTars and AgS-MULEs. In many cases examination of the structures flanking microsatellite targeted gSaTar elements reveals a lack TSDs, these insertion sites are thus inconsistent with insertion via the conventional MULE "cut and paste" mechanism (Fig. 4). The potential utilization of two distinct insertion mechanisms within gSaTar families

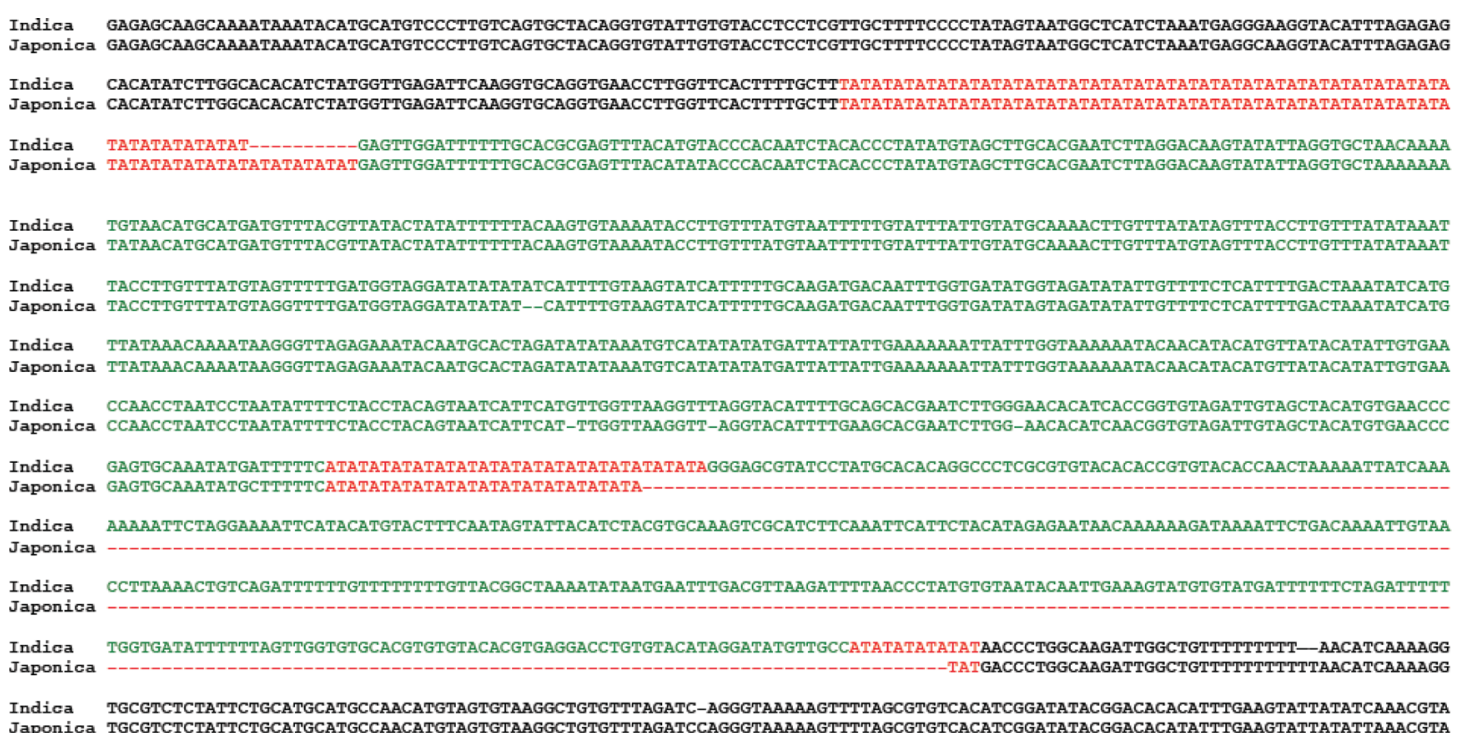

Fig. 8 Alignment of rice chromosome 1 sequences indicating an rSaTar3 insertion in Indica relative to Japonica. Microsatellite domains are indicated in red, gSaTar domains are indicated in green. Japonica sequence from Oryza sativa (japonica) v7_JGI 20451169-20452242. Indica from Oryza sativa (indica) assembly ASM465v1 22805255-22806717 
suggests the possibility element subtypes mobilized via independent pathways. However, probing (BLAST) the genome with either form (TSD or microsatellite targeted) returns equally elements with both types of insertion events, indicating that the two distinct insertion profiles do not appear to be associated with specific gSaTar subtypes. Rather, the gSaTar elements appear to be non-autonomous MULE elements that interact with, or are substrates of, an ancillary system that results in targeted insertion of the mobilized element into microsatellite domains.

The microsatellite targeting of gSaTar and AgS-MULE elements commonly results in clustering into mixedfamily multimers, within which individual elements are present in ordered, continuous arrays of uninterrupted elements. This structure feature was validated through PCR amplification of genomic regions containing gSaTar elements in guayule (Additional file 17). The expected nested architecture within these TE multimers, with older component elements interrupted by those more recently inserted [35, 44], was not observed in any of the gSatar- or AgS-MULE-containing clusters.

Given approximately 20,000 total members of the $g S a$ Tar families described here, a random distribution would result in approximately 1 element every $80 \mathrm{~kb}$. However, the observed distribution is strikingly non-random. Approximately $30 \%$ of the gSaTar elements from these families are linked directly to another gSaTar element. This value $(30 \%)$ represents a minimum level of association as our identification of gSaTar families is incomplete, and in many cases these elements are located on the scaffold ends where potential association would not be detected ( $\mathrm{gSaTar}$ elements are present at approximately $12 \%$ of scaffold ends). Given random insertion, the four linked elements shown in Additional file 3c would be expected to be distributed over approximately $320 \mathrm{~kb}$, not the observed $2.3 \mathrm{~kb}$.

Both the structure and distribution of the gSaTar and AgS-MULE elements suggest that while they share features with MULEs and Class II TEs in general, and can in cases be mobilized similarly, gSaTar transposition involves distinct and/or additional mobilization components. As such, they appear to serve as substrates in a novel mechanism that results in specific targeting to microsatellite domains and modular assembly of diverse elements at specific chromosomal loci.

\section{SaTar elements in other plant genomes}

To evaluate whether similar SaTars existed in other plant species, rice (Oryza sativa Japonica) was selected because of its well defined genome [37], the abundance and diversity of MULE elements [39], and the availability of genomic sequence from Oryza sativa Indica [40], a closely related subspecies/varietal group. The rice genome was analyzed employing a specific SaTar search algorithm (repetitive DNA sequences flanked by (TA) microsatellite domains) and five $r$ SaTar element families were returned (Table 3). The rSaTar elements were similar in size, structure and genomic distribution to the elements from guayule (Fig. 6, Table 3, Additional file 8). These elements could be classified as non-autonomous MULEs by sequence comparison (BLAST) to previously characterized TEs [36, 39], or by identification of an autonomous microsatellite targeted MULE with similar TIRs (Additional file 7). In addition, ancient autonomous, microsatellite-associated rice ArS-MULE elements present in $r S a T a r$ clusters were identified (Additional file 9).

Finally, SaTar families in sorghum (sSaTars, Additional files 13 and 14) and citrus (cSaTars, Additional files 15 and 16) were identified, suggesting that microsatellitetargeting dependent MULE stacking is a general feature of plant genomes. The relative similarity of SaTar family members varies considerably in the different species, for example the individual members of the cSaTar families have much lower similarity than observed among the gSaTars, indicating that SaTar targeting is evolutionarily intermittent.

\section{SaTar targeting}

The identification of SaTar elements in diverse plant genomic backgrounds offers the potential for an improved characterization of mobilization mechanisms. As discussed above, MULEs, as Class II TEs, are generally mobilized by a transposase-directed "cut and paste" mechanism [45] that results in TSDs, the size of which is diagnostic of the transposons superfamily. As indicated in Fig. 2, individual members of gSatar families not targeted to microsatellites are flanked by TSDs expected from $M u$-superfamily elements (8-11 bp TSDs in low-copy DNA), and are not assembled into arrays of unrelated elements joined end to end. The same is true of the non-targeted rSaTar3 elements (Table 3). In contrast, the sequences flanking many of the microsatellitetargeted gSaTar elements are inconsistent with the "cut and paste" mechanism (Fig. 4) suggesting involvement of a transpositional component distinct from other MULEs.

Given the general instability of microsatellite sequences [16], more recent SaTar insertion events would be expected to most accurately retain architectural features generated by the transposition. The TAFTA transposons in maize [20] represent the most recent events of MULE SaTar-like insertions described. In these events, empty target sites consisted of short TA microsatellite domains of (6-8 bp) which are expanded following insertion to up to $(\mathrm{TA})_{50}$ on each side of the inserted TAFTA element [20]. While similar apparent microsatellite expansions are easily found on comparison of relative $r S a T a r 2$ insertions in Japonica and Indica (Figs. 7 and 8, Additional file 12), the innate instability of the microsatellite domains, as well as 
inability to clearly differentiate $r S a T a r 2$ relative insertions and deletions, limits the utility of this data. In maize, a TA site-specific transposase was proposed to establish targeting, with expansion of the flanking microsatellite via DNA polymerase stuttering during repair of single stranded gaps [20]. However, in guayule (and other plant genomes, including rice) the SaTar-flanking microsatellite domains are often composed of diverse, non-TA, microsatellites (Fig. 4), complicating this targeting model. Rather, the targeting of SaTars to a diverse set of different microsatellite repeats suggests involvement of homologous recombination in SaTar insertion, not involved with insertion of non-SaTar MULE TEs. A separate model for the mechanism of SaTar targeting, and thus modular stacking of these TEs, would involve generation of closed circular SaTar intermediates [46] with microsatellite domains added as filler sequences between the element termini, followed by homologous recombination into an existing genomic microsatellite target domain. While this model is speculative in nature, the involvement of circular intermediates in MULE transposition has been suggested [12], and circular autonomous elements which include filler sequence have been described [47].

\section{SaTars and gene evolution}

While the actual importance of the MULE elements in the evolution of gene and genome architecture remains a topic of investigation [9], both the diversity and distribution of these elements in plant genomes suggests the potential to play an important role as mediators of plant gene evolution, including both coding and regulatory functions [12-15]. The specific targeting of these TEs to microsatellite domains, and the resulting modular assembly of mixed MULE clusters, offer improved evolutionary potential by preventing inactivation of active genes $[9,21]$ as well as proximally, and modularly, locating sequence divergent elements with the potential to contribute coding and non-coding gene components.

The SaTar elements described here do not represent a complete catalog of these elements in any of the selected genomes. However, the described elements are sufficient for demonstrating conservation of SaTar structure, chromosomal distribution profiles and cluster architecture among these plant species. It is clear that accurate determinations of both the frequency of SaTar clustering and overall contributions to these genomes will require more thorough characterization, and that the values presented here represent minimal estimates of their overall contributions to these genomes.

\section{Conclusion}

We report here the sequencing, assembly and annotation of the guayule genome to provide a foundation for application of modern crop improvement technologies to this plant. In addition, novel non-autonomous MULE SaTar elements with unique distribution profiles were identified in this genome, then characterized in other plant species. Satar targeting appears based on an alternative MULE recombination mechanism with the potential to impact gene evolution.

\section{Additional files}

\begin{abstract}
Additional file 1: gSaTar terminal inverted repeat sequences. (PDF $55 \mathrm{~kb}$ )
Additional file 2: gSaTar elements on the $16 \mathrm{Mb}$ artificial assembly (1\% of guayule genome). (PDF $41 \mathrm{~kb}$ )

Additional file 3: gSaTar clusters in the guayule CLC Genomics Workbench assembly. (PDF $46 \mathrm{~kb}$ )

Additional file 4: Autonomous gSaTar-MULE1 (AgS-MULE1) and cluster with non-autonomous gSaTar elements in the guayule CLC Genomics Workbench assembly. (PDF $47 \mathrm{~kb}$ )
\end{abstract}

Additional file 5: Alignment of non-autonomous gSaTar and autonomous gSaTar-MULE (AgS-MULE) terminal inverted repeat domains. (PDF 69 kb)

Additional file 6: Classification of rice rSaTar elements. (PDF 44 kb)

Additional file 7: The rSaTar2/Micron terminal inverted repeats are similar to those defining the ArS-MULE2 autonomous Mu-like transposon. (PDF $83 \mathrm{~kb}$ )

Additional file 8: Sample rSaTar clusters on the rice genome Oryza sativa V7_JGl. (PDF $45 \mathrm{~kb}$ )

Additional file 9: Autonomous rSaTar-Mules in the rice genome. (PDF $46 \mathrm{~kb}$ )

Additional file 10: Autonomous rSaTar-MULE1 (ArS-MULE1) element sequences in the rice (Japonica) genome. (PDF $140 \mathrm{~kb}$ )

Additional file 11: Alignment of rSaTar element conserved on Chromosome 1 of rice Japonica and Indica. (PDF 89 kb)

Additional file 12: Sample relative rSaTar insertions in Chromosome 1 of Oryza sativa Japonica and Indica. (PDF $45 \mathrm{~kb}$ )

Additional file 13: Architecture and distribution of sorghum sSaTar elements. (PDF $56 \mathrm{~kb}$ )

Additional file 14: sSatar cluster on sorghum Chromosome 1. (PDF 46 kb) Additional file 15: Architecture and distribution of citrus cSaTar elements. (PDF 59 kb)

Additional file 16: Sample cSaTar clusters on Citrus. (PDF 35 kb)

Additional file 17: $P C R$ amplification of genomic regions containing gSaTar clusters. (PDF $268 \mathrm{~kb}$ )

\section{Abbreviations}

MITEs: Miniature inverted-repeat transposable elements; MULE: Mu-like element; SaTar: Satellite Targeted; SSRs: Simple sequence repeats; TE: Transposable element

\section{Acknowledgements}

The authors thank Steven Huynh and Emma Yee for technical assistances in generating the guayule genomic sequence data. Mention of trade names or commercial products in this publication is solely for the purpose of providing specific information and does not imply recommendation or endorsement by the United States Department of Agriculture. The USDA is an equal opportunity provider and employer.

\section{Funding}

This work was funded and supported by the USDA-ARS (CRIS Project Numbers 2030-21220-001-00-D, 2030-21000-019-00-D and 2030-21410-021-00-D) and

USDA-NIFA/DOE Biomass Research and Development Initiative (BRDI) Grant No. 2012-10006. The funding agencies have not involved in the experimental design, analysis, and interpretation of the data or writing of the manuscript. 


\section{Availability of data and materials}

The raw sequence data for the guayule genome is deposited in NCBI under the accession number: SRP099139 and transcriptome data is deposited under the accession number: SRP116565. Other datasets used and/or analysed in this study are either available from the website: https://probes.pw.usda.gov/ Guayule/ or can be obtained upon request.

\section{Authors' contributions}

JAVF contributed to the guayule genome assembly and annotation, and drafting of the manuscript, and created the SaTar identification software; YW and $\mathrm{NH}$ contributed to acquisition of the guayule genome and transcriptome sequences, genome assembly and genome annotation; GP contributed to acquisition of the guayule transcriptome and drafting of the manuscript, HAC and CMM conceived of the project and contributed to drafting of the manuscript, YQG contributed to sequence acquisition, genome assembly and annotation, and drafting of the manuscript; WRB coordinated the project and contributed to bioinformatics analyses and drafting of the manuscript. All authors read and approved the final manuscript.

\section{Ethics approval and consent to participate}

The plant material was obtained from the USDA-ARS National Plant Germplasm System (NPGS; www.ars-grin.gov.npgs) through the National Arid Land Plant Genetics Recourses Unit (NALPGRU). Sampling of plant materials were performed in compliance with institutional, national, and international guidelines.

\section{Competing interests}

The authors declare that they have no competing interests.

\section{Publisher's Note}

Springer Nature remains neutral with regard to jurisdictional claims in published maps and institutional affiliations.

\section{Author details}

'Universidad Autónoma de Nuevo León, Monterrey, NL, Mexico.

USDA-Agricultural Research Service, Western Regional Research Center, Albany, CA, USA. ${ }^{3}$ Cooper Tire \& Rubber Company, Findlay, OH, USA. ${ }^{4}$ Present Address: Plant Breeding and Genetics Section, School of Integrative Plant Science, Cornell University, Ithaca, NY, USA.

\section{Received: 30 August 2017 Accepted: 10 April 2018}

\section{Published online: 19 April 2018}

\section{References}

1. Thompson AE, Ray DT. Breeding guayule. In: Plant Breeding Reviews. Hoboken: Wiley; 1989:93-165.

2. Ray DT, Foster MA, Coffelt TA, McMahan CM. Guayule: culture, breeding and rubber production. In: Singh BP, CAB International, editors. Industrial crops and uses; 2010. p. 384-410.

3. Board MR. Natural rubber statistics 2016; 2016. p. 1-26.

4. Lieberei R. South American leaf blight of the rubber tree (Hevea spp.): new steps in plant domestication using physiological features and molecular markers. Ann Bot. 2007;100(6):1125-42.

5. Ilut DC, Sanchez PL, Costich DE, Friebe B, Coffelt TA, Dyer JM, Jenks MA, Gore MA. Genomic diversity and phylogenetic relationships in the genus Parthenium (Asteraceae). Ind Crop Prod. 2015;76:920-9.

6. Sanchez PL, Costich DE, Friebe B, Coffelt TA, Jenks MA, Gore MA. Genome size variation in guayule and mariola: fundamental descriptors for polyploid plant taxa. Ind Crop Prod. 2014;54:1-5.

7. Yandell M, Ence D. A beginner's guide to eukaryotic genome annotation. Nat Rev Genet. 2012;13(5):329-42.

8. Kumar A, Bennetzen JL. Plant retrotransposons. Annu Rev Genet. 1999;33: 479-532.

9. Bennetzen $J$, Wang $H$. The contributions of transposable elements to the structure, function, and evolution of plant genomes. Annu Rev Plant Biol. 2014;65:505-30.

10. Lisch D. Mutator and MULE transposons. Microbiol Spectr. 2015;3(2):MDNA30032-2014.

11. Jiang N, Panaud O. Transposable element dynamics in rice and its wild relatives. In: Genetics and genomics of rice. Hoboken: Springer; 2013: 55-69.
12. Jiang N, Ferguson AA, Slotkin RK, Lisch D. Pack-Mutator-like transposable elements (Pack-MULEs) induce directional modification of genes through biased insertion and DNA acquisition. Proc Natl Acad Sci U S A. 2011;108(4): 1537-42.

13. Jiang N, Bao Z, Zhang X, Eddy SR, Wessler SR. Pack-MULE transposable elements mediate gene evolution in plants. Nature. 2004;431(7008):569-73.

14. Hanada K, Vallejo V, Nobuta K, Slotkin RK, Lisch D, Meyers BC, Shiu SH, Jiang $\mathrm{N}$. The functional role of pack-MULEs in rice inferred from purifying selection and expression profile. Plant Cell. 2009;21(1):25-38.

15. Lisch D. How important are transposons for plant evolution? Nat Rev Genet. 2013;14(1):49-61.

16. Ellegren H. Microsatellites: simple sequences with complex evolution. Nat Rev Genet. 2004;5(6):435-45.

17. Akagi H, Yokozeki Y, Inagaki A, Mori K, Fujimura T. Micron, a microsatellitetargeting transposable element in the rice genome. Mol Gen Genomics. 2001;266(3):471-80.

18. Temnykh S, DeClerck G, Lukashova A, Lipovich L, Cartinhour S, McCouch S Computational and experimental analysis of microsatellites in rice (Oryza sativa L.): frequency, length variation, transposon associations, and genetic marker potential. Genome Res. 2001;11(8):1441-52.

19. Tero N, Neumeier $H$, Gudavalli $R$, Schlotterer C. Silene tatarica microsatellites are frequently located in repetitive DNA. J Evol Biol. 2006;19(5):1612-9.

20. Wang Q, Dooner HK. Remarkable variation in maize genome structure inferred from haplotype diversity at the bz locus. Proc Natl Acad Sci U S A. 2006;103(47):17644-9.

21. Stawujak K, Startek M, Gambin A, Grzebelus D. MuTAnT: a family of Mutatorlike transposable elements targeting TA microsatellites in Medicago truncatula. Genetica. 2015;143(4):433-40.

22. Belknap WR, Wang Y, Huo N, Wu J, Rockhold DR, Gu YQ, Stover E. Characterizing the citrus cultivar Carrizo genome through 454 shotgun sequencing. Genome. 2011;54(12):1005-15.

23. Chapman JA, Ho I, Sunkara S, Luo S, Schroth GP, Rokhsar DS. Meraculous: de novo genome assembly with short paired-end reads. PLOS One. 2011; 6(8):e23501.

24. Boetzer M, Henkel CV, Jansen HJ, Butler D, Pirovano W. Scaffolding preassembled contigs using SSPACE. Bioinformatics. 2011;27(4):578-9.

25. Trapnell C, Williams BA, Pertea G, Mortazavi A, Kwan G, van Baren MJ, Salzberg SL, Wold BJ, Pachter L. Transcript assembly and quantification by RNA-Seq reveals unannotated transcripts and isoform switching during cell differentiation. Nat Biotechnol. 2010;28(5):511-5.

26. Grabherr MG, Haas BJ, Yassour M, Levin JZ, Thompson DA, Amit I, Adiconis X, Fan L, Raychowdhury R, Zeng Q, et al. Full-length transcriptome assembly from RNA-Seq data without a reference genome. Nat Biotechnol. 2011;29(7): 644-52.

27. Haas BJ, Delcher AL, Mount SM, Wortman JR, Smith RK Jr, Hannick LI, Maiti $\mathrm{R}$, Ronning CM, Rusch DB, Town CD, et al. Improving the Arabidopsis genome annotation using maximal transcript alignment assemblies. Nucleic Acids Res. 2003;31(19):5654-66

28. Cantarel BL, Korf I, Robb SM, Parra G, Ross E, Moore B, Holt C, Sanchez Alvarado A, Yandell M. MAKER: an easy-to-use annotation pipeline designed for emerging model organism genomes. Genome Res. 2008;18(1):188-96.

29. Barker MS, Kane NC, Matvienko M, Kozik A, Michelmore RW, Knapp SJ, Rieseberg LH. Multiple paleopolyploidizations during the evolution of the Compositae reveal parallel patterns of duplicate gene retention after millions of years. Mol Biol Evol. 2008;25(11):2445-55.

30. Yuan K, Wang C, Xin L, Zhang A, Ai C. Genomic organization and expression analysis of a farnesyl diphosphate synthase gene (FPPS2) in apples (Malus domestica Borkh.). Gene. 2013;524(2):90-4.

31. Nakamura A, Shimada H, Masuda T, Ohta H, Takamiya K. Two distinct isopentenyl diphosphate isomerases in cytosol and plastid are differentially induced by environmental stresses in tobacco. FEBS Lett. 2001;506(1):61-4.

32. Kim IJ, Ryu SB, Kwak YS, Kang H. A novel cDNA from Parthenium argentatum gray enhances the rubber biosynthetic activity in vitro. J Exp Bot. 2004;55(396):377-85.

33. Zhang Q, Arbuckle J, Wessler SR. Recent, extensive, and preferential insertion of members of the miniature inverted-repeat transposable element family heartbreaker into genic regions of maize. Proc Natl Acad Sci U S A. 2000;97(3):1160-5.

34. Mao L, Wood TC, Yu Y, Budiman MA, Tomkins J, Woo S, Sasinowski M, Presting G, Frisch D, Goff S, et al. Rice transposable elements: a survey of 73,000 sequence-tagged-connectors. Genome Res. 2000;10(7):982-90. 
35. Jiang N, Wessler SR. Insertion preference of maize and rice miniature inverted repeat transposable elements as revealed by the analysis of nested elements. Plant Cell. 2001;13(11):2553-64.

36. Copetti D, Zhang J, El Baidouri M, Gao D, Wang J, Barghini E, Cossu RM, Angelova A, Maldonado LC, Roffler S, et al. RiTE database: a resource database for genus-wide rice genomics and evolutionary biology. BMC Genomics. 2015;16:538

37. Ouyang S, Zhu W, Hamilton J, Lin H, Campbell M, Childs K, Thibaud-Nissen $F$, Malek RL, Lee Y, Zheng L, et al. The TIGR rice genome annotation resource: improvements and new features. Nucleic Acids Res. 2007; 35(Database issue):D883-7.

38. Kawahara Y, de la Bastide M, Hamilton JP, Kanamori H, McCombie WR, Ouyang S, Schwartz DC, Tanaka T, Wu JZ, Zhou SG, et al. Improvement of the Oryza sativa Nipponbare reference genome using next generation sequence and optical map data. Rice. 2013;6:4

39. Ferguson AA, Zhao D, Jiang N. Selective acquisition and retention of genomic sequences by pack-Mutator-like elements based on guaninecytosine content and the breadth of expression. Plant Physiol. 2013;163(3): 1419-32.

40. Yu J, Hu S, Wang J, Wong GK, Li S, Liu B, Deng Y, Dai L, Zhou Y, Zhang X, et al. A draft sequence of the rice genome (Oryza sativa L. ssp. indica). Science. 2002:296(5565):79-92.

41. Ma J, Bennetzen JL. Rapid recent growth and divergence of rice nuclear genomes. Proc Natl Acad Sci U S A. 2004;101(34):12404-10.

42. Paterson AH, Bowers JE, Bruggmann R, Dubchak I, Grimwood J, Gundlach H, Haberer G, Hellsten U, Mitros T, Poliakov A, et al. The Sorghum bicolor genome and the diversification of grasses. Nature. 2009;457(7229):551-6.

43. Wu GA, Prochnik S, Jenkins J, Salse J, Hellsten U, Murat F, Perrier X, Ruiz M, Scalabrin S, Terol J, et al. Sequencing of diverse mandarin, pummelo and orange genomes reveals complex history of admixture during citrus domestication. Nat Biotechnol. 2014;32(7):656-62.

44. SanMiguel P, Tikhonov A, Jin YK, Motchoulskaia N, Zakharov D, MelakeBerhan A, Springer PS, Edwards KJ, Lee M, Avramova Z, et al. Nested retrotransposons in the intergenic regions of the maize genome. Science. 1996:274(5288):765-8.

45. Yuan YW, Wessler SR. The catalytic domain of all eukaryotic cut-and-paste transposase superfamilies. Proc Natl Acad Sci U S A. 2011;108(19):7884-9.

46. Sundaresan V, Freeling M. An extrachromosomal form of the Mu transposons of maize. Proc Natl Acad Sci U S A. 1987:84(14):4924-8.

47. Li Y, Harris L, Dooner HK. TED, an autonomous and rare maize transposon of the mutator superfamily with a high gametophytic excision frequency. Plant Cell. 2013;25(9):3251-65.

\section{Ready to submit your research? Choose BMC and benefit from:}

- fast, convenient online submission

- thorough peer review by experienced researchers in your field

- rapid publication on acceptance

- support for research data, including large and complex data types

- gold Open Access which fosters wider collaboration and increased citations - maximum visibility for your research: over $100 \mathrm{M}$ website views per year 